\title{
Elimination of Mother-to-Child Transmission of HIV — Thailand
}

\author{
Rangsima Lolekha, $\mathrm{MD}^{1}$; Sarawut Boonsuk, $\mathrm{MD}^{2}$; Tanarak Plipat, MD, PhD³ ; Michael Martin, $\mathrm{MD}^{1}$; Chaweewan Tonputsa, $\mathrm{MA}^{2}$; \\ Niramon Punsuwan, MS 3 ; Thananda Naiwatanakul, MSC ${ }^{1}$; Kulkanya Chokephaibulkit, MD ${ }^{4}$; Hansa Thaisri, MSC ${ }^{5}$; Praphan Phanuphak, MD, PhD ${ }^{6}$; \\ Suchada Chaivooth, $\mathrm{MD}^{7}$; Sumet Ongwandee, $\mathrm{MD}^{3}$; Benjamas Baipluthong, $\mathrm{MPH}^{1}$; Wachira Pengjuntr, $\mathrm{MD}^{2}$; Sopon Mekton, $\mathrm{MD}^{8}$
}

Thailand experienced a generalized human immunodeficiency virus (HIV) epidemic during the 1990s. HIV prevalence among pregnant women was $2.0 \%$ and the mother-to-child transmission (MTCT) rate was $>20 \%(1-3)$. In June 2016, Thailand became the first country in Asia to validate the elimination of MTCT by meeting World Health Organization (WHO) targets. Because Thailand's experience implementing a successful prevention of MTCT program might be instructive for other countries, Thailand's prevention of MTCT interventions, outcomes, factors that contributed to success, and challenges that remain were reviewed. Thailand's national prevention of MTCT program has evolved with prevention science from national implementation of short course zidovudine (AZT) in 2000 to lifelong highly active antiretroviral therapy regardless of CD4 count (WHO option B+) in 2014 (1). By 2015, HIV prevalence among pregnant women had decreased to $0.6 \%$ and the MTCT rate to $1.9 \%$ (the elimination of MTCT target is $<2 \%$ for nonbreastfeeding populations) (4). A strong public health infrastructure, committed political leadership, government funding, engagement of multiple partners, and a robust monitoring system allowed Thailand to achieve this important public health milestone.

\section{Early prevention of MTCT response}

The first case of HIV in a pregnant woman in Thailand was reported in 1988 and increasing HIV prevalence among pregnant women and other populations was recognized in the early 1990s (3,5). In 1996, after the ACTG 076 trial* (6), the Thailand Ministry of Public Health (MOPH) and Siriraj Hospital, in collaboration with CDCThailand/Southeast Asia Regional Office, launched a trial of short-course oral AZT, a regimen feasible for use in Thailand (2). The trial demonstrated a $50 \%$ reduction in MTCT.

In 1996, Her Royal Highness Princess Soamsawali donated funds to the Thai Red Cross Society to make antiretrovirals for prevention of MTCT available to hospitals around the country. During 1997-1999, the MOPH implemented pilot prevention of MTCT projects in northeastern (7) and northern Thailand (5) to provide HIV testing for pregnant women and AZT for

\footnotetext{
*ACTG 076 was a Phase III, randomized, double-blind, placebo-controlled clinical trial designed to evaluate whether zidovudine administered orally (initiated at 14-34 weeks gestation) and intravenously during labor to HIVinfected pregnant women and orally to their infants could reduce the rate of transmission from mother to infant.
}

prevention of MTCT, and to implement a pilot prevention of MTCT monitoring system. In 2000, the Department of Health (DOH) $\mathrm{MOPH}$ announced the first national prevention of MTCT policy and issued guidelines for all government hospitals to integrate prevention of MTCT activities into routine maternal and child health services, including HIV testing for all pregnant women, antiretroviral therapy for prevention of MTCT, and infant formula for infants born to HIV-positive mothers. The prevention of MTCT program covers all public and private health care facilities. The Thai government funds prevention of MTCT services for Thais under the universal health coverage policy. During 2007-2014, non-Thai HIV-positive pregnant women could access prevention of MTCT services through a Global Fund project; these services can currently be accessed through hospital social welfare funds, the Princess Soamsawali prevention of MTCT fund, government-sponsored migrant health insurance, or other special projects (1) (Figure 1).

Antiretroviral regimens for Thailand's national prevention of MTCT program have evolved with prevention science. In 2000, HIV-positive pregnant women were offered AZT starting at 34 weeks gestation and their infants received AZT for 4 weeks. A single-dose of nevirapine (WHO option A) was added in 2004; next, in 2010, highly active antiretroviral therapy (WHO option $\mathrm{B}$ ) was provided during pregnancy and continued based on CD4 count; and finally, in 2014, highly active antiretroviral therapy for life regardless of CD4 count (WHO option $\mathrm{B}+$ ) became the standard. HIV testing of couples was implemented in 2010 (1).

Infant HIV testing guidelines have also evolved. During 2000-2006, HIV diagnosis in infants aged 12 months and 18 months was accomplished using antibody tests; diagnoses in some infants aged $>2$ months were made using DNA polymerase chain reaction (PCR) testing as part of research studies or other projects. In 2007, HIV DNA PCR testing was implemented for infants aged 1-2 months and 2-4 months using national HIV/AIDS funds. In 2014, the national prevention of MTCT guidelines were modified to classify infants based on their risk for acquiring HIV. Infants with standard risk receive AZT for 4 weeks, and HIV DNA PCR testing is performed at age 1 month and 2-4 months. Infants with high risk (maternal plasma HIV viral load $>50$ copies/mL or infants born to mothers taking highly active antiretroviral therapy for $<4$ weeks before delivery) receive AZT, lamivudine, and nevirapine for 
FIGURE 1. Timeline of the prevention of mother-to-child transmission (MTCT) of HIV policy — Thailand, 1993-2015

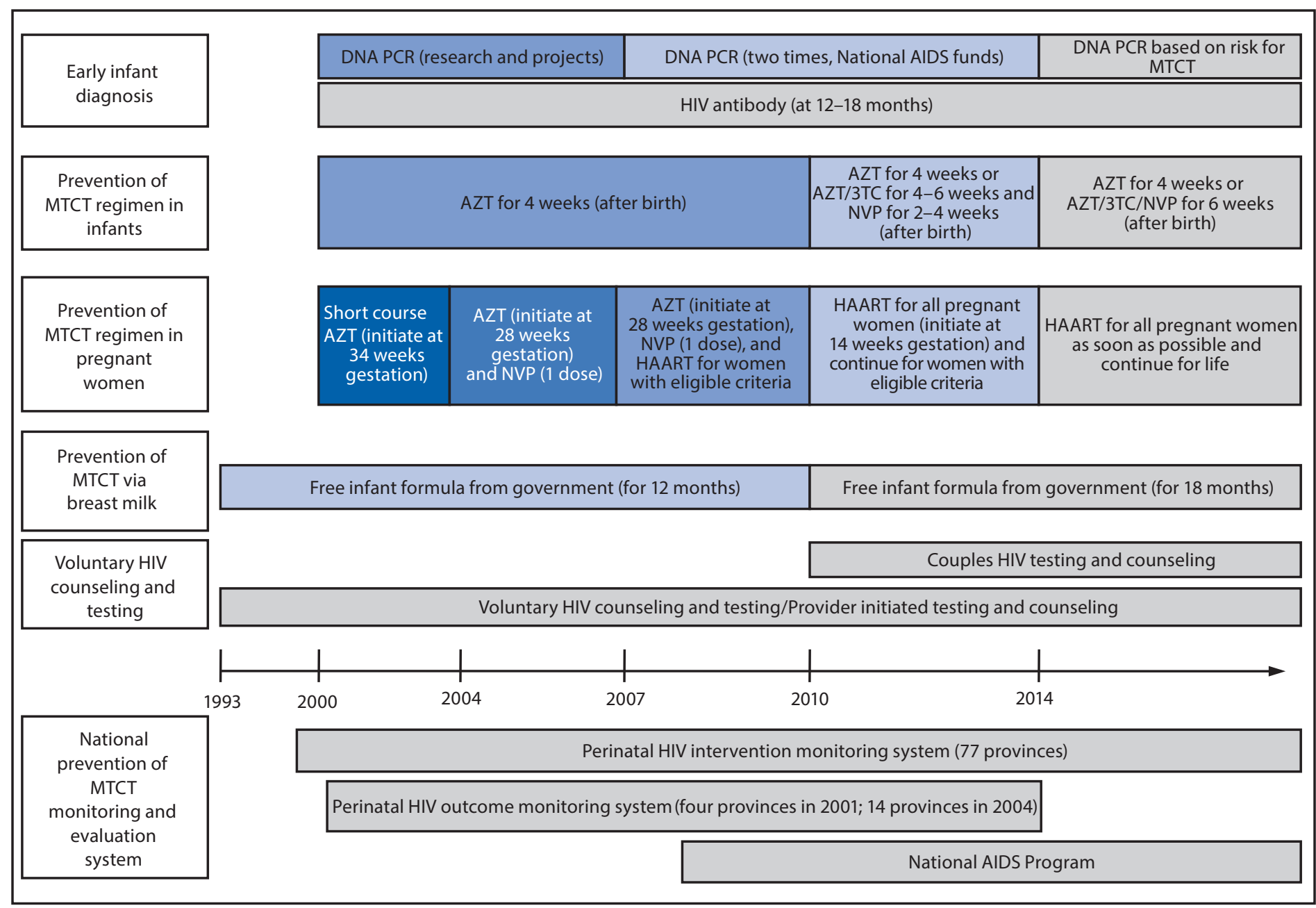

Abbreviations: $3 \mathrm{TC}=$ lamivudine; AIDS = acquired immunodeficiency syndrome; $\mathrm{AZT}=$ zidovudine; HAART = highly active antiretroviral therapy; HIV = human immunodeficiency virus; NVP = nevirapine; $P C R=$ polymerase chain reaction.

6 weeks, and HIV DNA PCR testing is performed at ages 1 , 2, and 4 months. All children born to HIV-positive mothers have confirmatory HIV antibody testing at age 18 months (1).

Stigma and discrimination against women living with HIV continues to prevent some women from accessing antenatal clinic services (1). Women living with HIV in Thailand and civil society organizations have worked with the MOPH to develop and implement a training curriculum for hospital personnel that aims to reduce stigma and discrimination (I).

\section{National prevention of MTCT monitoring system}

In 2000, the DOH MOPH, with assistance from CDC, launched the Perinatal HIV Intervention Monitoring System (PHIMS) to monitor prevention of MTCT services (8). PHIMS collects monthly summaries from hospitals, including HIV testing of pregnant women and their partners, and antiretroviral coverage for prevention of MTCT. PHIMS has been integrated in routine hospital reporting activities, and in 2015, PHIMS covered 837 (92\%) governmental hospitals in Thailand (77\% of total deliveries including Thais and non-Thais).

Thailand has high levels of health care coverage: $98.3 \%$ of pregnant women had at least one antenatal clinic visit in 2015 (elimination of MTCT target $>95 \%$ ) (4). The percentage of pregnant women tested for HIV has increased from 61.9\% among women in the 1998 prevention of MTCT pilot projects (7) to $92.9 \%$ in 2001(8) after the national prevention of MTCT policy was announced, and to $99.6 \%$ in 2015 (elimination of MTCT target $>95 \%)(1)$. The use of antiretrovirals for prevention of MTCT increased from 64.6\% in 1998 ( 7 ) to $71.4 \%$ in 2001 (8), and to $95.6 \%$ in 2015 (elimination of MTCT target $>90 \%$ ) (1) (Table). 
Morbidity and Mortality Weekly Report

TABLE. Coverage of prevention of mother-to-child transmission (MTCT) of HIV services using Perinatal HIV Intervention Monitoring System (PHIMS) data for Thai and non-Thai populations - Thailand, July 1998-June 1999, 2001, 2005, 2011, and 2015

\begin{tabular}{|c|c|c|c|c|c|}
\hline \multirow[b]{2}{*}{ Indicator (definition) } & \multicolumn{5}{|c|}{ Reporting time frame } \\
\hline & $\begin{array}{c}\text { July } 1998- \\
\text { June } 1999^{*} \\
\text { No. }(\%) \\
\text { (n=774,349') }\end{array}$ & $\begin{array}{c}2001^{\S} \\
\text { No. }(\%) \\
\left(n=766,107^{\dagger}\right)\end{array}$ & $\begin{array}{c}2005^{\emptyset} \\
\text { No. }(\%) \\
\left(\mathrm{n}=822,593^{\dagger}\right)\end{array}$ & $\begin{array}{c}2011^{* *} \\
\text { No. }(\%) \\
\left(n=796,091^{\dagger}\right)\end{array}$ & $\begin{array}{c}2015^{\dagger \dagger} \\
\text { No. }(\%) \\
\left(n=736,352^{\dagger}\right)\end{array}$ \\
\hline Coverage of reporting governmental hospitals & $7(-)^{\S \S}$ & 793/853 ตึๆ $(93.0)$ & $804 / 893$ กีศ $(90.0)$ & $487 / 868$ ๆๆ $(56.1)^{* * *}$ & 837/914 ๆๆ (91.6) \\
\hline Deliveries covered by PHIMS ${ }^{+\dagger}$ & $75,308(9.7)$ & $653,576(85.3)$ & $692,133(84.1)$ & $364,455(45.8)$ & $566,403(76.9)$ \\
\hline Pregnant women receiving antenatal care ${ }^{\S \S}$ & $74,511(98.9)$ & $631,344(96.6)$ & $678,565(98.0)$ & $356,532(97.8)$ & $556,773(98.3)$ \\
\hline Coverage of pregnant women tested for HIV§§§ & $46,648(61.9)$ & $607,336(92.9)$ & $688,955(99.5)$ & $363,848(99.8)$ & $564,125(99.6)$ \\
\hline Pregnant women testing HIV positive & $410(0.88)$ & $7,659(1.26)$ & $6,231(0.90)$ & $2,333(0.64)$ & $3,399(0.60)$ \\
\hline $\begin{array}{l}\text { HIV-positive pregnant women receiving ART for } \\
\text { prevention of } \mathrm{MTCT} \S \S \S\end{array}$ & $265(64.6)$ & $5,466(71.4)$ & $5,584(89.6)$ & 2,191 (93.9) & 3,249 (95.6) \\
\hline Live infants born to HIV-positive pregnant women & - & $7,492(97.8)$ & $6,037(96.9)$ & $2,274(97.5)$ & $3,385(99.6)$ \\
\hline HIV-exposed infants who received ART for PMTCT & - & $6,718(89.7)$ & $5,961(98.7)$ & $2,238(98.4)$ & $3,368(99.5)$ \\
\hline Partners tested for HIV & - & - & - & - & $239,473(42.3)$ \\
\hline Partners testing HIV positive & - & - & - & - & $1,003(0.4)$ \\
\hline
\end{tabular}

\footnotetext{
Abbreviations: ART = antiretroviral therapy; HIV = human immunodeficiency virus.

* Data from pilot project in region 7 (Northeastern Thailand). Kanshana S, Thewanda D, Teeraratkul A, et al. Implementing short-course zidovudine to reduce motherinfant HIV transmission in a large pilot program in Thailand. AIDS. 2000 Jul 28;14(11):1617-23.

† Total number of deliveries in Thailand. Data from Ministry of Interior.

$\S$ First year data from PHIMS report; 1 year after the national prevention of MTCT policy launched in 2000. 2001 represents October 2000-September 2001 based on Thailand governmental reporting practice; a similar time-frame was used for 2005, 2011, and 2015.

I PHIMS data 1 year after WHO option A Prevention of MTCT Policy implemented in Thailand in 2004.

** PHIMS data 1 year after WHO option B Prevention of MTCT Policy implemented in Thailand in 2010.

t† PHIMS data 1 year after WHO option B+ Policy implemented in Thailand in 2014.

$\S \S$ Seven provinces in Region 7 (Northeastern Thailand).

१ी Number of hospitals reporting/number of hospitals covered by PHIMS.

*** Coverage of PHIMS report was low because of the transition of the PHIMS system from a local network-based system to a web-based system.

${ }^{+t+}$ Number of women reported in PHIMS (\% of total deliveries).

$\S \S \S$ WHO targets for elimination of MTCT of HIV: antenatal care coverage (at least one visit) $\geq 95 \%$; HIV testing coverage of pregnant women $\geq 95 \%$; ART coverage of HIV-positive pregnant women $\geq 90 \%$.
}

The MOPH Bureau of Epidemiology, with support from CDC, launched the Perinatal HIV Outcome Monitoring System in 2001 (9). Providers in 64 public hospitals in four of the country's 77 provinces submitted data, including the number of infants born to HIV-positive mothers, the number of HIV-infected infants, and the MTCT rate, to the Perinatal HIV Outcome Monitoring System, which expanded to 191 facilities in 14 provinces during 2004-2007. In 2008, Thailand established the National AIDS Program to monitor national HIV treatment and care services. MTCT rates were calculated based on infant HIV DNA PCR test results reported in the National AIDS Program. Adjusted MTCT rates during 2001-2012 were calculated to include HIV-exposed infants who were not tested for HIV or whose HIV test results were not reported (9,10). During 2013-2015, adjusted MTCT rates were calculated using SPECTRUM version 5.4 (1).

With the implementation of HIV prevention policies and increased coverage of effective prevention tools (e.g., HIV testing and antiretrovirals for prevention of MTCT) and strong prevention of MTCT monitoring systems, the MTCT rate decreased from $24.2 \%$ in 1994 (2) to $10.2 \%$ in 2003 with the introduction of short-course AZT, to $4.5 \%$ with the implementation of WHO option A, and to $1.9 \%$ in 2015 after the implementation of WHO option B+ (Figure 2).

\section{Discussion}

Thailand has achieved WHO targets for the elimination of MTCT, and is the first country with a generalized HIV epidemic to reach this milestone. The prevalence of HIV among pregnant women has decreased substantially during the past two decades. A combination of factors has made this possible. The Thai government responded to the increasing prevalence of HIV among pregnant women by working with domestic and international medical experts and researchers to assess available data, initiate studies where needed, build the capacity of health care workers, launch national HIV education and $100 \%$ condom use campaigns, implement pilot prevention of MTCT activities, gather evidence to develop national policy, and expand activities nationwide. The government also engaged with civil society, persons living with HIV, and nongovernmental organizations to consider appropriate and feasible prevention interventions. A well-developed national health and laboratory system, the integration of prevention of MTCT into routine maternal child health care, and government funding of prevention of MTCT services have been 
FIGURE 2. Rate of mother-to-child transmission (MTCT) of HIV* and timeline for introduction of MTCT prevention regimens ${ }^{\dagger}-$ Thailand $^{*}$ 2001-2015§

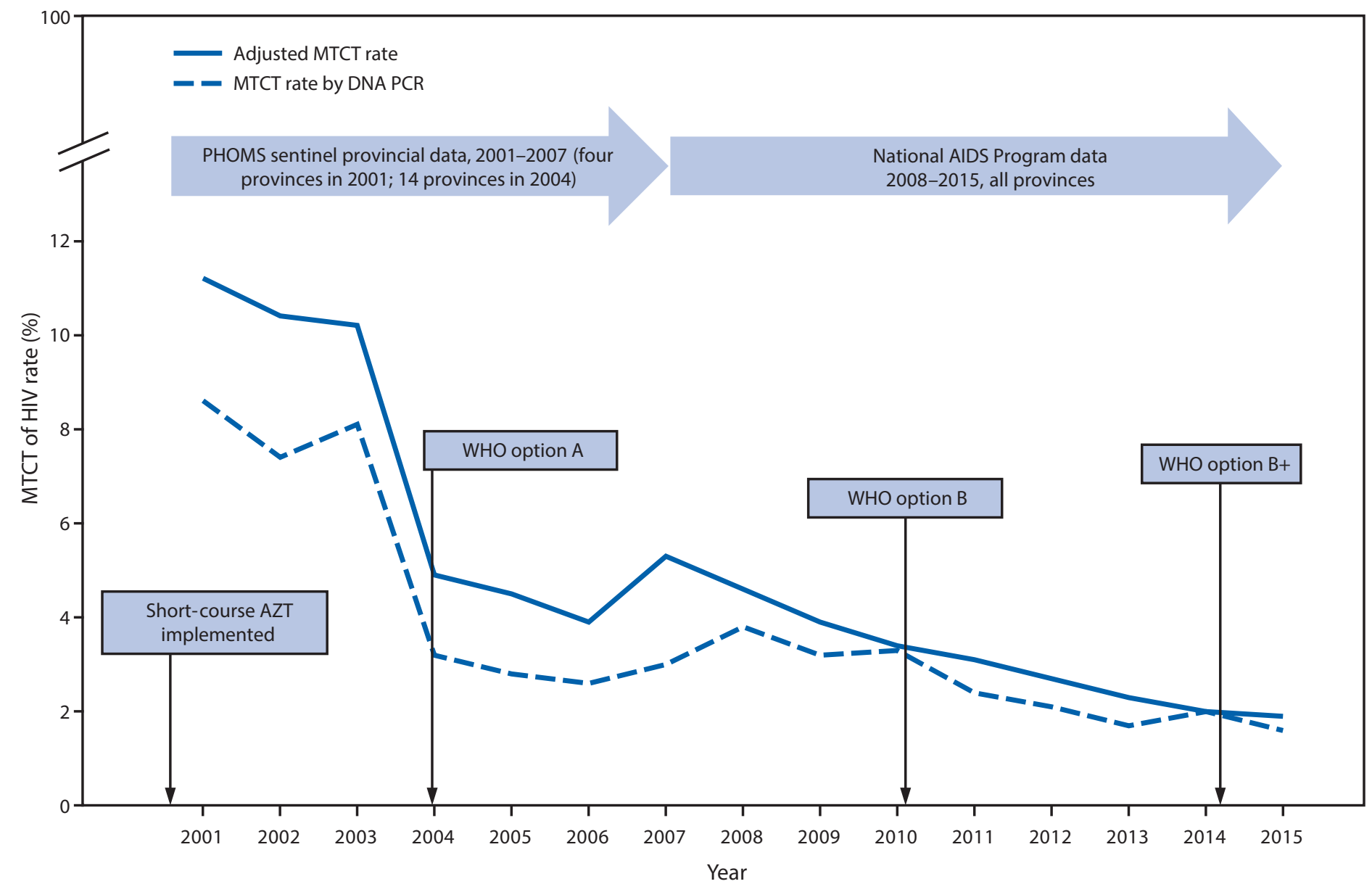

Abbreviations: AIDS = acquired immunodeficiency syndrome; AZT = zidovudine; HIV = human immunodeficiency virus; $P C R=$ polymerase chain reaction; PHOMS = Perinatal HIV Outcome Monitoring System, WHO = World Health Organization.

* The adjusted MTCT rates during 2001-2012 were calculated to include HIV-exposed infants who were not tested for HIV or whose HIV test results were not reported (http://dx.doi.org/10.1097/QAD.0b013e328010e02d; http://dx.doi.org/10.7448/IAS.19.1.20511). The adjusted MTCT rates during 2013-2015 were calculated using SPECTRUM version 5.4 (a software tool developed by the Joint United Nations Programme on HIV/AIDS and partners to assist countries in monitoring their HIV epidemic and provide outputs such as the number of pregnant women and infants infected with HIV). The MTCT rate was calculated based on national infant HIV DNA PCR test results.

${ }^{\dagger}$ In 2000, HIV-positive pregnant women were offered AZT starting at 34 weeks gestation and their infants received AZT for 4 weeks. A single-dose of nevirapine (WHO option A) was added in 2004; next, in 2010 highly active antiretroviral therapy (WHO option B) was provided during pregnancy and continued based on CD4 count; and finally, highly active antiretroviral therapy for life regardless of CD4 count (WHO option B+) became the standard in 2014.

$\S$ 2001-2007: method of calculation for estimates of MTCT rate described at http://dx.doi.org/10.1097/QAD.0b013e328010e02d; 2008-2012: global AIDS response report 2008-2012; 2013-2015: SPECTRUM version 5.4.

important in attaining high coverage and consistent prevention of MTCT services nationwide. Thailand has a robust national prevention of MTCT monitoring and evaluation system that promotes data use for program improvement at national and subnational levels. As a result, the expanding epidemic of HIV among women was stemmed and MTCT reduced, and fewer infants are born HIV-positive in Thailand.

The findings in this report are subject to at least three limitations. First, nationwide surveillance data about HIV testing coverage and the MTCT rate in the 1980s and 1990s are lacking. Second, assessments of HIV testing and the MTCT rate did not cover $23 \%$ of deliveries in 2015. Finally, the DOH sent a prevention of MTCT coverage questionnaire during 2013-2015 to 170 hospitals that are not part of the PHIMS reporting system, including 140 private hospitals, 19 non$\mathrm{MOPH}$ government hospitals, and 11 university hospitals; although only $39 \%$ responded, coverage of antenatal clinics, HIV testing, and antiretrovirals for prevention of MTCT met elimination of MTCT targets in the hospitals that responded.

Thailand's national AIDS strategy aims to reduce the MTCT rate to $<1 \%$ by 2030 . Preliminary data from an active case management network launched in Thailand in August 2014 
suggested that approximately $80 \%$ of new perinatal HIV cases occurred among women who begin antenatal clinic services late, have poor antiretroviral therapy adherence, or test HIVnegative at the first antenatal clinic visit but acquire HIV later (before or after delivery) (1). In response, Thailand's National HIV Treatment and Prevention Guideline 2016 will recommend raltegravir, an integrase inhibitor with rapid antiviral activity, for HIV-positive pregnant women who receive care after 32 weeks of pregnancy, and emphasize HIV testing of couples beginning during visits to antenatal clinics and continuing through the postpartum period. Data suggest that to reach a MTCT rate $<1 \%$, Thailand will need to strengthen ownership of prevention of MTCT at subnational and community levels, enhance prevention of MTCT monitoring and data use, ensure that HIV-positive migrants have access to HIV services; and sustain the active case management system.

\section{Acknowledgments}

Siriporn Kanchana, MD, Nipunporn Voramongkol, MD, Pornsinee Amornwichet, Nareeluck Kullerk, Thailand Department of Health; technical specialists from 12 regional health promotion centers; Robert James Simonds, MD, Achara Teeraratkul, MD, CDC; Busarawan Sriwanthana, PhD, DMSc, Nittaya Phanuphak, MD, PhD, Thai Red Cross AIDS Research Center; Sorakij Bhakeecheep, MD, National Health Security Office, Thailand; Tanawan Samleerat, PhD, Chiang Mai University; staff members of the Active Case Management Network Working Group; staff members of the Thailand SPECTRUM Working Group; Division of Global HIV/AIDS and TB, CDC; Division of HIV/AIDS Prevention, CDC; PEPFAR; health care workers, program managers, volunteers, persons living with HIV, leaders both in the health facilities and the communities.

\footnotetext{
${ }^{1}$ CDC Thailand/Southeast Asia Regional Office, Nonthaburi, Thailand; ${ }^{2}$ Department of Health, Ministry of Public Health, Nonthaburi, Thailand; ${ }^{3}$ Department of Disease Control, Ministry of Public Health, Nonthaburi, Thailand; ${ }^{4}$ Department of Pediatrics, Siriraj Hospital, Mahidol University, Bangkok, Thailand; 5 Department of Medical Sciences, Ministry of Public Health, Nonthaburi, Thailand; ${ }^{6}$ Thai Red Cross AIDS Research Center, Bangkok, Thailand; ${ }^{7}$ The Thailand National Health Security Office, Nonthaburi, Thailand; ${ }^{8}$ Thailand Ministry of Public Health, Nonthaburi, Thailand.
}

Corresponding author: Rangsima Lolekha, hpu8@cdc.gov, 66-2-580-0669.

\section{References}

1. Thailand Ministry of Public Health. Validation of elimination of motherto-child transmission of HIV and syphilis, Thailand 2013-2015 Report. Nonthaburi, Thailand: Thailand Ministry of Public Health; 2016.

2. Shaffer N, Chuachoowong R, Mock PA, et al.; Bangkok Collaborative Perinatal HIV Transmission Study Group. Short-course zidovudine for perinatal HIV-1 transmission in Bangkok, Thailand: a randomised controlled trial. Lancet 1999;353:773-80. http://dx.doi.org/10.1016/ S0140-6736(98)10411-7

3. Bunnell RE, Yanpaisarn S, Kilmarx PH, et al. HIV-1 seroprevalence among childbearing women in northern Thailand: monitoring a rapidly evolving epidemic. AIDS 1999;13:509-15. http://dx.doi. org/10.1097/00002030-199903110-00010

\section{Summary}

What is already known about this topic?

Thailand experienced a generalized human immunodeficiency virus (HIV) epidemic in the 1990s. HIV prevalence among women in antenatal clinics was $2 \%$, and mother-to-child transmission (MTCT) rate of HIV was $>20 \%$.

What is added by this report?

Thailand has achieved World Health Organization targets for the elimination of MTCT. With implementation of programs for $100 \%$ condom use and HIV prevention, HIV prevalence among pregnant women decreased from $2 \%$ in the mid-1990s to $0.6 \%$ in 2015. The MTCT rate decreased from $>20 \%$ to $1.9 \%$ because of the effective use of antiretroviral regimens to prevent MTCT, including the adoption of WHO option $\mathrm{B}+$ (lifelong highly active antiretroviral therapy regardless of CD4 count) in 2014, and the high coverage of antenatal care and prevention of MTCT services in Thailand. Factors that contributed to these achievements include the commitment and leadership of the Thai government, a strong public health infrastructure, a self-reliant national budget, the engagement of nongovernmental and civil society partners, and a robust prevention of MTCT monitoring program.

What are the implications for public health?

Thailand has achieved World Health Organization elimination of MTCT targets and can serve as a model for other countries.

4. World Health Organization. Elimination of mother-to-child transmission (EMTCT) of HIV and syphilis. Global guidance on criteria and processes for validation. Geneva, Switzerland: World Health Organization; 2014. http://www.who.int/hiv/pub/emtct-validation-guidance/en/

5. Thaineua V, Sirinirund P, Tanbanjong A, Lallemant M, Soucat A, Lamboray JL. From research to practice: use of short course zidovudine to prevent mother-to-child HIV transmission in the context of routine health care in Northern Thailand. Southeast Asian J Trop Med Public Health 1998;29:429-42.

6. Connor EM, Sperling RS, Gelber R, et al. Reduction of maternal-infant transmission of human immunodeficiency virus type 1 with zidovudine treatment. Pediatric AIDS Clinical Trials Group Protocol 076 Study Group. N Engl J Med 1994;331:1173-80. http://dx.doi.org/10.1056/ NEJM199411033311801

7. Kanshana S, Thewanda D, Teeraratkul A, et al. Implementing shortcourse zidovudine to reduce mother-infant HIV transmission in a large pilot program in Thailand. AIDS 2000;14:1617-23. http://dx.doi. org/10.1097/00002030-200007280-00018

8. Amornwichet P, Teeraratkul A, Simonds RJ, et al. Preventing motherto-child HIV transmission: the first year of Thailand's national program. JAMA 2002;288:245-8. http://dx.doi.org/10.1001/jama.288.2.245

9. Plipat T, Naiwatanakul T, Rattanasuporn N, et al. Reduction in motherto-child transmission of HIV in Thailand, 2001-2003: Results from population-based surveillance in six provinces. AIDS 2007;21:145-51. http://dx.doi.org/10.1097/QAD.0b013e328010e02d

10. Naiwatanakul T, Voramongkol N, Punsuwan N, et al. Uptake of early infant diagnosis in Thailand's national program for preventing motherto-child HIV transmission and linkage to care, 2008-2011. J Int AIDS Soc 2016;19:20511. http://dx.doi.org/10.7448/IAS.19.1.20511 